\title{
Gestão de resíduos nos estados/municípios brasileiros: avaliação e análise dos procedimentos para a escolha do modelo de gestão ambiental
}

\author{
Mamadu Lamarana Bari? \\ Henrique Tomé da Costa Mata ${ }^{2}$ \\ Lívio Andrade Wanderley ${ }^{3}$
}

Resumo: O artigo visa identificar indicadores apropriados de valor econômico e de gestão socioambiental de resíduos sólidos urbanos e rurais, considerando as respectivas unidades de medição e os métodos apropriados. Nesse sentido, foi utilizado o método de análise multivariada, onde inicialmente se empregou a análise fatorial para determinar as relações quantitativas entre as variáveis chaves do estudo e ordená-las de acordo com os fatores causais. Em seguida, aplicou-se a análise de cluster, para separar os estados/municípios em grupos homogêneos segundo o sistema de coleta e destinação dos resíduos. Os resultados obtidos foram satisfatórios por terem permitido identificar grupos de estados com idênticas similaridades na gestão dos resíduos.

Palavras Chaves: Resíduos. Modelo de Gestão. Análise Multivariada.

1 Professor Adjunto da Faculdade de Economia da Universidade Federal de Mato Grosso (UFMT)

2 Professor Associado e vice-diretor da Faculdade de Economia da Universidade Federal da Bahia (UFBA)

3 Professor Associado e Coordenador do Programa de Pós-Graduação em Economia/UFBA 


\begin{abstract}
The article aims to identify appropriate indicators of economic value and environmental management of municipal solid waste and rural, where as the corresponding units of measurement and the appropriate methods. In this sense, has used the method of multivariate analysis, where initially used Factor Analysis to determine the quantitative relationships between the key variables of the study and sorts them according to the causal factors. Then it was applied to the analysis of Cluster, to separate the states/cities in homogeneous groups according to the system of collection and disposal of waste. The results were satisfactory for having allowed identify groups of states with identical similarities in waste management.
\end{abstract}

Key Words: Waste. Management Model. Multivariate Analysis.

\title{
I Introdução
}

\section{I.I Problema e sua importância}

A produção dos resíduos sólidos está na ordem direta do crescimento populacional e do grau de urbanização e da extensão da fronteira agrícola. Segundo a pesquisa publicada pelo Instituto Brasileiro de Geografia e Estatística (IBGE, 2011), constata-se que taxa de crescimento médio da população no ano de 2010 é de 1,17, e das agriculturas é de 1,4\%. Isso, aliado à taxa de crescimento da população urbana e da produção agrícola, passa a constituir um problema na produção e destinação final dos resíduos sólidos.

Baseado nesses fatos, pode-se afirmar que quanto maior é a taxa de urbanização e das atividades agrícolas, maior é o crescimento do uso de bens que produzem resíduos tanto urbano como proveniente de descarte das embalagens de agrotóxicos. Nesse sentido, a mesma pesquisa revela que cerca de $68,5 \%$ dos resíduos produzidos pelas populações da maioria dos municípios são depositados em lixões e em terrenos alagados e, a partir dessas constatações, afirma-se que maiores problemas socioambientais poderão ser gerados quando mal administrados os resíduos urbanos. A gestão trata do manejo do ciclo dos resíduos sólidos, que compreende: a geração, coleta (tradicional e seletiva), tratamento e disposição final.

Sabe-se que a gestão de resíduos sólidos não engloba apenas aspectos da natureza de serviços de coleta, mas também aspectos institucionais, administrativos operacionais e políticos. Por muito tempo, o objeto da gestão de resíduos sólidos se resumia à coleta, ao tratamento e à eliminação 
de resíduos. A partir do RIO 92, as denúncias feitas sobre o mau uso e destinação dos resíduos estavam pondo em perigo a vida na Terra, por isso foram feitas algumas recomendações que foram transformadas em estratégias para o desenvolvimento sustentável, dentre as quais se destacam a minimização dos resíduos, a reutilização/reaproveitamento e reciclagem.

Hoje, a discussão que se trava no que se refere às atividades de lavoura está centrada em visão maniqueísta sobre tipos de atividades agrícolas praticadas nos estados ou municípios. Ou seja, tudo o que é relativo à agricultura familiar é visto como atividade agrícola limpa, portanto, é celestial. E aquelas atividades relativas à grande agricultura são vistas como sujas e poluidoras ambientais, logo, endemoniadas. Nessa base, o princípio corretamente útil para a lavoura e para a saúde humana consiste na busca de uma nova concepção de práticas de produção e de consumo, conhecida como tecnologias limpas, ou seja, na adoção do método de coleta seletiva, triagem e reciclagem dos resíduos sólidos. A valorização dos recicláveis contidos nos resíduos sólidos urbanos - o valor econômico extraído dos resíduos - não é uma atividade recente, pois ela ocorre de forma institucionalizada através de programas de coleta seletiva municipal, de usinas de reciclagem (resíduos não segregados em sua fonte de origem) e do trabalho de populações marginalizadas, catadores de papel e/ou catadores em lixões conhecidos, como "badameiros" (catadores do lixo).

Tabela 1 - Estimativa da composição gravimétrica dos resíduos sólidos urbanos coletados no Brasil em 2008

\begin{tabular}{c|c|c}
\hline Resíduos & Participação (\%) & Quantidade (t/dia) \\
\hline Material Reciclável & $\mathbf{3 1 , 9}$ & $\mathbf{5 8 . 5 2 7 , 4 0}$ \\
\hline Metais & 2,9 & $5.293,50$ \\
Aço & 2,3 & $4.213,70$ \\
Alumínio & 0,6 & $1.079,90$ \\
Papel, papelão, etc. & 13,1 & $23.997,40$ \\
Plástico total & 13,5 & $24.847,90$ \\
Plástico filme & 8,9 & $16.399,60$ \\
Plástico rígido & 4,6 & $8.448,30$ \\
Vidro & 2,4 & $4.388,60$ \\
Emb. Agro-tóxicos & 12,0 & $22.023,00$ \\
Matéria orgânica & 51,4 & $94.335,10$ \\
Outros & 16,7 & $30.618,90$ \\
Total & 100,0 & $183.481,50$ \\
\hline
\end{tabular}

Fonte: Brasil.

Plano Nacional de Resíduos Sólidos. Brasília-DF. 2011.

Segundo Bursztyn (2000), o aumento do volume e do valor dos resíduos urbanos (consequência direta da evolução da sociedade industrializada) promove o crescimento preocupante da população excluída ou 
marginalizada, o qual estimula a formação de verdadeiros "batalhões" de pessoas que vivem dentro dos lixões.

No que se refere apenas ao uso e descarte de embalagens de agrotóxicos, vale considerar os dados apresentados pelo Instituto Nacional de Processamentos de Embalagens Vazias (InpEV), que representa a indústria fabricante de agrotóxicos para a destinação das embalagens vazias de seus produtos. De acordo com dados do INPEV (2010), 12 estados apresentaram crescimento no volume destinado quando comparados aos mesmos meses de 2011. Os estados que tiveram maior destaque foram: Mato Grosso (5.959 toneladas), São Paulo (2.530 toneladas), Goiás (2.356 toneladas), Rio Grande do Sul (2.061 toneladas), Minas Gerais (1.827 toneladas), Bahia (1.757 toneladas) e Mato Grosso do Sul (1.536 toneladas), que, juntos, respondem por $78 \%$ do volume total destinado em todo o país.

Ainda a análise revela que os estados que alcançaram os maiores percentuais de crescimento no período foram: Rio de Janeiro, com 295\% (de 13 para 52 toneladas); Sergipe, com 106\% (de 11 para 22 toneladas); e Mato Grosso, com 30\% (de 4.580 para 5.959 toneladas).

Tabela 2 - Produção dos resíduos sólidos por estados no Brasil (2010-2011)

\begin{tabular}{c|c|c|c}
\hline Estado & Volume 2010 (t) & Volume 2011 (t) & Crescimento (\%) \\
\hline Mato Grosso & 4.580 & 5.959 & 30 \\
\hline São Paulo & 2.336 & 2.530 & 8 \\
\hline Goiás & 2.236 & 2.356 & 24 \\
\hline Rio Grande do Sul & 1.666 & 2.061 & 11 \\
\hline Minas Gerais & 1.643 & 1.827 & 23 \\
\hline Bahia & 1.435 & 1.757 & 24 \\
\hline Mato Grosso do Sul & 1.487 & 1.536 & 4 \\
\hline Maranhão & 363 & 450 & 16 \\
\hline Santa Catarina & 316 & 327 & 295 \\
\hline Piauí & 157 & 182 & 106 \\
\hline Rio de Janeiro & 13 & 52 & -15 \\
\hline Sergipe & 11 & 22 & 12 \\
\hline Outros & 3.490 & 2.961 & 22.023 \\
\hline Brasil & 19.730 & & 3 \\
\hline
\end{tabular}

Fonte: InpEV. (2010) 
Nos últimos 10 anos, segundo InpEV (2010), mais de 237 mil toneladas de embalagens de agrotóxicos utilizadas nas propriedades rurais brasileiras voltaram para os fabricantes, que reutilizaram ou eliminaram o material, seguindo padrões ambientais definidos em lei. Entretanto, ainda que medidas-esforços sejam empreendidas para que se impacte menos o solo com o descarte de embalagens de agrotóxico, os dados revelados nesta pesquisa mostram a necessidade de um estudo mais aprofundado, que subsidie as políticas públicas dos governos nas esferas municipal, estadual e federal sobre o destino e tratamento dos resíduos urbanos. É nesse contexto que o presente artigo visa identificar procedimentos mais apropriados para gestão socioambiental de resíduos sólidos urbanos, considerando as respectivas unidades de coleta e os métodos apropriados.

A análise e avaliação de procedimentos apropriados para manejo de resíduos municipais permitirá ao poder público maior segurança para melhorar ou escolher seu modelo de gestão em função dos resultados revelados no trabalho.

\section{Modelo conceitual}

\section{I Critérios para seleção dos indicadores}

O programa de indicadores ambientais da Organização de Cooperação e Desenvolvimento Econômicos (OCDE) reconhece que não existe um conjunto universal de indicadores, mas vários conjuntos, correspondendo, cada um deles, a objetivos específicos. Podem ser empregados em níveis internacional e nacional para estabelecer relatórios sobre o estado do meio ambiente, examinar os desempenhos ambientais e relatar os progresso alcançados em matéria de desenvolvimento sustentável. E podem, ainda, servir, em nível nacional, para o planejamento, o esclarecimento dos objetivos políticos e a definição das prioridades.

A escolha do modelo adequado para a gestão dos resíduos não é fácil de conceber se levarmos em consideração a precariedade das informações sobre a produção e coleta dos resíduos sólidos urbanos. Essa preocupação parte da constatação e análise da natureza dos serviços de limpeza e/ou coleta de lixo nos municípios do Brasil, bem como da destinação final do lixo coletado.

A partir do momento que o objetivo deste trabalho é identificar o empenho de municípios no que respeita a adoção de sistema apropriada 
de coleta e destino do lixo urbano, a fim de impactar menos o meio ambiente, bem como preservar os mananciais dos rios e solos adjacentes aos lixões, então qualquer tomada de decisão deve estar ligada a implementações de políticas públicas em direção a esse problema. Assim, a sugestão do modelo de aferição dos serviços de saneamento nos municípios deve contemplar não apenas os agentes econômicos envolvidos nessa prática e a relação de troca que se realiza, mas também considerar os riscos ambientais e de saúde que os resíduos provocam quando mal administrados.

A partir dessas constatações, propõe-se analisar os problemas dos resíduos sólidos urbanos utilizando o método de análise multivariada. Usa-se análise fatorial para determinar as relações quantitativas entre as variáveis chaves do estudo e ordená-las de acordo com os fatores causais. Esse método visa designar os fatores que indicarão o grau de adequação ou de eficiência de cada estabelecimento em termos de produção e coleta de resíduos urbanos, ou seja, quando os resíduos recebem tratamento na destinação final (triagem, reciclagem, compostagem ou incineração). E, ainda, usar-se-á a análise cluster para testar, ou melhor, constatar, a qualidade de agregação das variáveis realizadas pela análise fatorial. Desse modo, a análise fatorial ajuda a avaliar a coleta do lixo em termos de adequação ou inadequação segundo o destino final. Considera-se como estudo o sistema inadequado - quando o destino final da coleta não obedece aos critérios apontados.

\section{Metodologia}

A metodologia adotada para determinar a escolha e análise dos indicadores é baseada no método de análise multivariada. O software utilizado para a estimação e análise dos dados pelo método citado é o SPSS versão 20.0. Os dados foram coletados em uma amostra de 27 estados do Brasil. O período da coleta corresponde ao ano de 2011, e as variáveis escolhidas foram selecionadas do censo do IBGE - Pesquisa Nacional do Saneamento Básico 2011.

Da metodologia proposta, seleciona-se, inicialmente, as técnicas de análise fatorial para determinar as relações quantitativas entre as variáveis e ordená-las de acordo com os fatores causais. Para assegurar a veracidade das relações estabelecidas, isto é, se a qualidade da agregação de variáveis realizada pela análise fatorial pode ser testada, recorre-se às técnicas de análise de cluster ou de discriminante. 


\section{I Análise Fatorial}

A análise fatorial é uma técnica estatística usada para identificar, em termos relativos, um pequeno número de fatores que podem ser usados para indicar as relações estatísticas entre um conjunto de variáveis inter-relacionadas.

A pressuposição básica da análise fatorial é de que existem certos fatores causais gerais que originam as correlações observadas entre as variáveis em estudo. O número desses fatores é sempre menor que o das variáveis que compõem a série, pois a maior parte das variáveis relacionadas é explicada pelos mesmos fatores causais.

No modelo de análise fatorial, cada uma das variáveis $j X(j=1,2, . ., p)$ pode ser escrita como uma combinação linear dos fatores comuns $(\mathrm{F} 1, \mathrm{~F} 2, \ldots, \mathrm{Fm})$, que explica a parcela da variância de cada variável explicada pelos fatores comuns mais um desvio que resume a parcela da variância total não explicada pelos fatores comuns. A parcela explicada pelos fatores comuns recebe o nome de comunalidade, e a parcela não explicada pelos fatores comuns o de especificidade.

As comunalidades podem variar de 0 a 1 ; as que se aproximam de zero (0) indicam que os fatores comuns não explicam a variância, e as que se aproximam de um (1) indicam que todas as variâncias são explicadas pelos fatores comuns, demonstrando, com isso, um bom ajustamento do modelo.

Algebricamente, o modelo pode ser representado como segue:

$$
X j=\lambda 1 j F 1+\lambda 2 j F 2+\ldots+\lambda 1 m F m+e j ; \quad j=1,2, \ldots, p
$$

O parâmetro $\lambda$ ij é denominado carga fatorial (factor loading) e reflete a importância do fator $i$ na explicação da variável $j$. Os $F$ são os fatores, e o erro é designado por e.

$\mathrm{Na}$ forma matricial, o sistema pode ser representado desta maneira:

$$
X=F A+U
$$

onde $X$ é vetor das variáveis originais; $F$ é o vetor dos fatores comuns; $A$ é a matriz de "conexão", ou seja, contém as cargas fatoriais, e $U$ é o vetor de fatores específicos.

A questão central da análise fatorial reside, pois, em determinar a matriz fatorial reduzida, denominada matriz da carga fatorial (reduzida) $F_{B}=|f j m| n x r$. Essa matriz revela, então, as relações entre as variáveis normalizadas $\mathrm{Zi}$ e os fatores comuns, abandonando-se os específicos e os fatores de erro. (GONTIJO; AGUIRRE, 1988) 
Para se chegar a esses resultados, a análise fatorial é feita a partir da análise da variância, concretamente, da matriz das correlações entre as variáveis, $\mathrm{RA}=\mid$ rih $\mid$

Considerando as premissas usuais, tem-se que

$$
\begin{aligned}
& F i \sim N(0,1) \text { e, são independentes } \\
& \text { ej N } \sim(0,1) \text { e, são independentes } \\
& \text { Fi e ej são independentes entre si } \\
& E(x j)=0
\end{aligned}
$$

A condição de ortogonalidade entre os fatores, entre os erros e os fatores, e a independência dos erros, levando em consideração a premissa (5), pode ser expressa por

$$
\begin{aligned}
& \sigma_{j}^{2}=\lambda l j^{2}+\lambda 2 j^{2}+\ldots+\lambda m j^{2}+\Psi j \\
& \text { onde } \sigma_{j}^{2} \text { é a variância de } \mathrm{Xj} .
\end{aligned}
$$

Assim, a variância total da variável Xj pode ser decomposta numa parcela explicada pelo modelo e denominada comunalidade e uma parcela não explicada, denominada especificidade.

$$
V(x j)=\lambda 1 j^{2}+\lambda 2 j^{2}+\ldots .+\lambda m^{2} j+\Psi j=\stackrel{m}{a}^{m} \mid i^{2}
$$

Portanto, $\square$ j é a especificidade, enquanto as parcelas anteriores são as comunalidades. A comunalidade e a especificidade de $\mathrm{Xj}$ devem somar 1. Para se avaliar o grau de adequação do modelo, deve-se observar se as comunalidades das variáveis são próximas de 1. Quanto mais próximas da unidade estiverem, melhor é o ajustamento do modelo. Da mesma forma, pode-se dizer que, quanto menor for a especificidade, melhor é o modelo.

Tabela 3 - O Grau de Ajustamento do Modelo Econométrico pelas Cumunalidades das Variáveis

\begin{tabular}{c|c}
\hline Variáveis & Comunalidades \\
\hline Coleta & 0,993 \\
\hline Seletiva & 0,962 \\
\hline Reciclagem & 0,993 \\
\hline Compostagem & 0,913 \\
\hline Incineração & 0,999 \\
\hline Composição & 1,000 \\
\hline
\end{tabular}

Fonte: dados da pesquisa 
A Tabela 3 resume para cada variável a comunalidade obtida. Verifica-se que todas elas apresentam coeficiente próximo à unidade, por exemplo: para a variável tecnológica RECICLA, os fatores comuns explicam cerca de $99,3 \%$ da variância total, a diferença percentual da parcela não explicada é especificidade.

As cargas fatoriais finais referem-se à correlação que existe entre cada fator e cada uma das variáveis, ou seja, elas mostram a importância de cada fator na explicação de cada uma das variáveis e podem ser obtidas através da rotação de fatores. Existem vários métodos de rotação de fatores. Neste trabalho, adotou-se o método varimax de rotação ortogonal, pois tal método tenta minimizar o número de variáveis que têm alta carga fatorial. Os resultados obtidos são apresentados na Tabela 4.

Tabela 4 - A Matriz dos Fatores Rodados

\begin{tabular}{c|c|c}
\hline & Fator 1 & Fator 2 \\
\hline Coleta & 0,397 & 0,866 \\
\hline Selet & 0,916 & 0,222 \\
\hline Recicla & 0,920 & 0,264 \\
\hline Compst & 0,832 & 0,408 \\
\hline Incin. & 0,563 & 0,379 \\
\hline Compos. & 0,108 & 0,109 \\
\hline
\end{tabular}

Fonte: dados da pesquisa.

As cargas fatoriais obtidas representam a correlação entre cada fator e cada uma das variáveis, ou seja, a importância de cada fator na explicação de cada uma das variáveis. Por exemplo, tem-se que corr. ( $F 1$, Recicla $=0,920)$.

Segundo se pode observar na Tabela 4, a correlação entre fatores e variáveis encontram-se separadas em dois blocos.

O fator 1 explica o comportamento das variáveis SELET, RECICLA e COMPOST. Esse fator indica o nível adequado do tratamento dos resíduos sólidos dentro dos padrões socioambientais.

O fator 2 apresenta apenas uma variável, a COLETA e indica o nível inadequado do tratamento dos resíduos na destinação final.

Tabela 5 - Fatores que Explicam as Variações da Coleta e

Tratamento dos Resíduos Sólidos urbanos

\begin{tabular}{c|c|c|c}
\hline Fator & Autovalores & \% das var & \% acum \\
\hline 1 & 4,279 & 71,31 & 71,31 \\
\hline 2 & 0,923 & 15,39 & 86,70 \\
\hline
\end{tabular}

Fonte: dados da pesquisa 
Na Tabela 5 pode-se notar que o fator 1 explica cerca de 71,31\% da variância total dos dados sobre a destinação final dos resíduos sólidos, enquanto o fator 2 explica cerca de 15,39\%. Conjuntamente, explicam cerca de $86,70 \%$ da variância total. A parcela restante da variância total 13,3\% não explicada pelo modelo deve-se à especificidade.

A substituição de seis componentes originais por dois fatores representa uma aproximação boa, uma vez que o modelo explica cerca de $86,70 \%$ da variância total dos dados. Por outro lado, considerando-se que os fatores substituem de forma satisfatória as variáveis originais, pode-se então obter uma ordenação dos estados/municípios levando em conta apenas esses fatores.

Para se obter uma classificação de acordo com cada um dos fatores, é necessário o alcance da matriz dos coeficientes fatoriais. Esses coeficientes são obtidos multiplicando-se a matriz transposta das cargas fatoriais pela inversa da matriz da correlação:

$$
C F=\square^{*} R^{-1}
$$

onde, $\square$ 'é a inversa da matriz de cargas fatoriais,

$\mathrm{R}^{-1}$ é a inversa da matriz da correlação.

Os dados estão representados na Tabela 4.

Tabela 6 - Os Escores da Matriz dos Coeficientes Fatoriais

\begin{tabular}{c|c|c}
\hline Variáveis & Fator 1 & Fator 2 \\
\hline COLETA & $-0,255$ & 1,458 \\
\hline SELET & 0,768 & $-0,121$ \\
\hline RECICLA & 0,692 & $-0,110$ \\
\hline COMPST & $-0,028$ & $-0,170$ \\
\hline INCIN & $-0,397$ & $-0,349$ \\
\hline COMPOS & $-0,097$ & $-0,165$ \\
\hline
\end{tabular}

Fonte: dados da pesquisa.

A partir dos coeficientes fatoriais, pode-se estimar os escores fatoriais através da seguinte equação:

$$
F=\square^{*} R^{-1 * X}
$$

onde, $\square$ matriz transposta das cargas fatoriais; 
$\mathrm{R}^{-1}$ inversa da matriz de correlação original;

$\mathrm{X}$ matriz dos dados originais padronizados;

F matriz das estimativas dos "scores" fatoriais.

Para cada estado/município, estimou-se os escores fatoriais. Através desses escores, foi possível classificá-los numa escala que acompanha a distribuição em ordem decrescente com relação ao uso do fator. Cada estado/município está representado através de um número cadastral, e o trabalho compõe-se de 27 amostras.

A cada estado/município foi atribuído o valor alto ou médio do fator. Aquele que se situar no primeiro intervalo de acordo com a classificação estabelecida significa que apresenta alto valor do fator, se ficar situado no segundo intervalo, apresenta valor médio do fator.

Os escores fatoriais mostram que em determinados períodos houve estados/municípios que se situaram em uma ou outra faixa do intervalo, isso pode refletir problemas quanto à gestão dos resíduos, quanto à natureza de serviços de coleta e na forma de destinação final.

O grau de adequação, ou melhor, a eficiência de cada estabelecimento em termos de produção e coleta será analisada em cada fator tomando como parâmetro dois tipos de classificação: alto e médio. Os intervalos serão construídos com base no limite superior da distribuição (maior valor observado), e no limite inferior (menor valor observado), assim: o limite máximo corresponde a 4,3166 e o limite mínimo corresponde a-0,66411 do fator 1. Para o fator 2, esses limites são de 3,9000 a - 1,1507.

\begin{tabular}{|c|c|c|}
\hline & Fator 1 & Fator 2 \\
\hline Alto & 4,3166 a 1,2981 & 3,9000 a 1,5366 \\
\hline Médio & 1,2981 a $-0,6641$ & 1,5366 a $-1,1507$ \\
\hline
\end{tabular}

No fator 1, os estados/municípios com sistema de coleta adequado somam cerca de $11,11 \%$ do total de 27 observações e cerca de $88,9 \%$ têm sistema de coleta, embora na classe de adequado, mas com alguns sistemas de tratamento precário. No que se concerne ao fator 2 , cerca de $7,4 \%$ dos estado/municípios se encaixam na faixa alta, ou seja, estão em fase de transição, em busca do melhoramento do tratamento dos resíduos sólidos, e cerca de 92,6\% estão na faixa dos que não usam nenhum sistema de tratamento de resíduos. 


\subsection{Análise cluster}

Deve-se ressaltar que o método de análise fatorial faz uma seleção importante e ajuda a interpretar as relações que surgem de cada fator separado. Segundo Gontijo e Aguirre (1988), cada escolha e cada interpretação é, em maior ou menor medida, subjetiva, por isso, esse modelo é passível de crítica. Depois de se obter o resultado da análise, não se pode assegurar que as relações estabelecidas sejam únicas e verdadeiras. Levando em conta essas considerações, torna-se aconselhável recorrer ao uso da técnica de agrupamentos (cluster) para testar a qualidade da agregação das variáveis realizadas pela análise fatorial. Como o objetivo do trabalho é agrupar os estados/municípios com o mesmo sistema de coleta e destinação dos resíduos sólidos urbanos, escolheu-se, então, para o teste o método cluster de casos.

O objetivo principal da análise, nesta seção, será o estudo da similaridade entre os estados/municípios, tendo como base os dois fatores. O primeiro conjunto congrega os estados/municípios como o melhor sistema de coleta e destinação dos resíduos e corresponde ao fator 1 . O segundo conjunto é composto de estados/municípios com sistema de coleta fora das recomendações ambientais e corresponde ao fator 2 .

Os resultados são mostrados no dendrograma, e o método usado foi o Método de Ligação Simples (MLS) ou denominado de vizinho mais próximo. Esse método define como similaridade entre dois grupos aquela dada pelos dois membros mais parecidos. Ou seja, entre todos os coeficientes de similaridade dos elementos de um grupo e de outro, escolhe-se o de maior similaridade como o coeficiente entre dois grupos. (BUSSAB; MIAZAKI; ANDRADE, 1990) Assim, dados os conjuntos de objetos X e Y, a distância entre eles será definida como:

$$
d(X, Y)=\min (d i j: i \in X e j \in Y)
$$

Essas distâncias são obtidas quantitativamente pela raiz quadrada da distância euclidiana:

$$
d^{*}(X, Y)=\sqrt{\stackrel{a}{a}_{k=1}^{p}(X i K-Y j K)^{2}}
$$




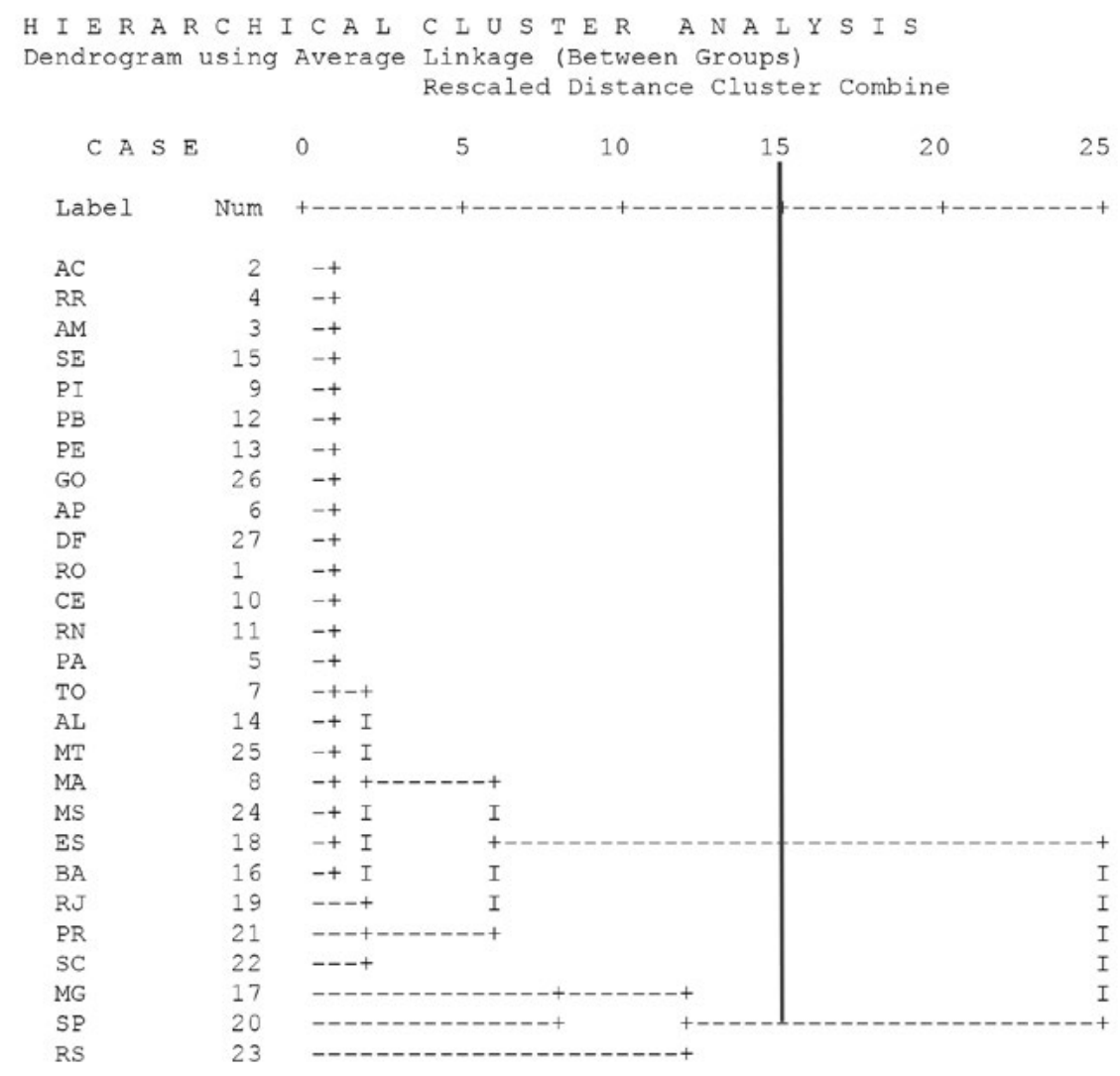

Desse modo, observando-se o gráfico, pode-se afirmar que a análise cluster de casos confirma de forma satisfatória o resultado da análise fatorial. Selecionando o gráfico na escala 15 da distância euclidiana, têm-se as combinações dos casos em dois grupos, dado que se optou pela distância mínima. O terceiro grupo não chega a ser descartado por se encontrar incluído no segundo grupo, mas dada a expressividade do grupo dois, ele ficou absorvido por este grupo, o que significa dizer que existe certa similaridade no modo de gestão dos resíduos sólidos. Assim, podem ser vistos os estados $7,14,25,8,24,18,16,19,21$ e 22 no mesmo grupo ou cluster, e os estados 17, 20 e 23 fazendo parte de outro grupo. O primeiro grupo corresponde ao fator 1 , e o segundo grupo ao fator 2 .

Os estados/municípios foram classificados por estrutura hierárquica de similaridade através do escore fatorial, onde poderão ser agrupados em 
dois intervalos de classes distintos. O cluster de casos procurará formá-los em grupos homogêneos e diferenciados entre si.

De acordo com o MLS utilizado, pelo que se constatam no dendrograma de casos, os estados/municípios apresentam-se divididos em dois grupos homogêneos, onde a maioria se encontra no mesmo grupo e apenas quatro fazendo parte de outro grupo isolado. Além da conformidade com o método usado, esse caso pode ser justificado também pela própria característica do sistema de coleta e destinação final dos resíduos urbanos dos estados/municípios estudados.

\section{Conclusão}

Os resultados obtidos indicam que o método de análise multivariada se adéqua para a análise de escolha de indicadores de gestão dos estados/ municípios estudados e, também, para agrupar os estados similares segundo o grau de adequação ou inadequação no sistema de tratamento dos resíduos urbanos.

A aplicação da análise fatorial permitiu a redução de seis variáveis/ indicadores do sistema de coleta, quanto à destinação final dos resíduos sólidos urbanos, em dois fatores, que foram satisfatoriamente confirmados pela análise cluster de casos.

A análise de cluster de casos permitiu não só agrupar os estados/ municípios segundo suas similaridades, mas também identificá-los quanto ao grau de adequação ou não do sistema de coleta e destinação final dos resíduos sólidos.

\section{Referências}

BARI, M. L.; GOMES, A. P. Tipificação de produtores de leite através da análise multivariada. In: CONGRESSO BRASILEIRO DE ECONOMIA E SOCIOLOGIA RURAL DA SOBER, 34.,1996, Aracaju. Anais... Viçosa, MG: SOBER, 1996.

BARROS, R. T de V. Saneamento. In: MANUAL de Saneamento e proteção ambiental para municípios. Belo Horizonte: Ed. UFMG, Departamento de Engenharia, 1995.

BRASIL. Ministério do Meio Ambiente. Plano Nacional de Resíduos Sólidos. Brasília-DF: Governo Federal: Ministério do Meio Ambiente, 2011. (Versão preliminar). 
BURSZTYN, M. No meio da rua: nômades, excluídos e viradores. Rio de Janeiro: Garamond, 2000. 261 p.

BUSSAB, W. O.; MIASAKI, E. S.; ANDRADE, D. F. de. Introdução à análise de agrupamentos. In: SIMPÓSIO BRASILEIRO DE PROBABILIDADE ESTATÍSTICA, 9., 1990, São Paulo. Anais... São Paulo: ABE: USP, 1990.

EVERITT, B. Cluster Analysis. London: Social Science Research Council: HEB, 1977. $122 \mathrm{p}$.

FABRIANI, C. B.; PEREIRA, V. M. C. Tendências e divergências sobre o modelo de intervenção pública no saneamento básico. Rio de Janeiro: UFRJ: IEI, 1987.

GONTIJO, C.; AGUIRRE, A. Elementos para uma tipologia do uso do solo agrícola no Brasil: uma aplicação de análise fatorial. Revista Brasileira de Economia. Rio de Janeiro, v. 42, n. 1, p. 13-49, jan./mar.1988.

IBGE - Instituto Brasileiro de Geografia e Estatística. Pesquisa Nacional de Saneamento Básico - PNSB, 2011. Rio de Janeiro: IBGE, Departamento de Estatística e Indicadores Sociais. 2011.

INPEV - Instituto Nacional de Processamento de Embalagens Vazias. Relatório de sustentabilidade 2010. São Paulo: InpEV, 2010. Disponível em: < http:// www.inpev.org.br/educacao/publicacoes/relatorio_anual/2010/port/download/ Inpev-2010>. Acesso em: 19 mar. 2013.

KLEINBAUM, D. G.; KUPPER, L. Applied Regression Analysis And Other Multivariable Methods. Massachussetts: Duxbury Press, 1978. 556 p.

NUNESMAIA, M. F. Gestion de déchets urbains socialement intégrée: le cas Brésil. 2001. 276 p. Tese Doutorado) - École doctorale Sciences et Ingénierie, Université Cergy-Pontoise, France, 2001.

PFEIFFER, D. Disparidades de desenvolvimento no Brasil: um exemplo da análise cluster. Revista Brasileira de Estatística, Rio de Janeiro, v. 41, n. 164, p. 559-576, out./dez. 1980. 


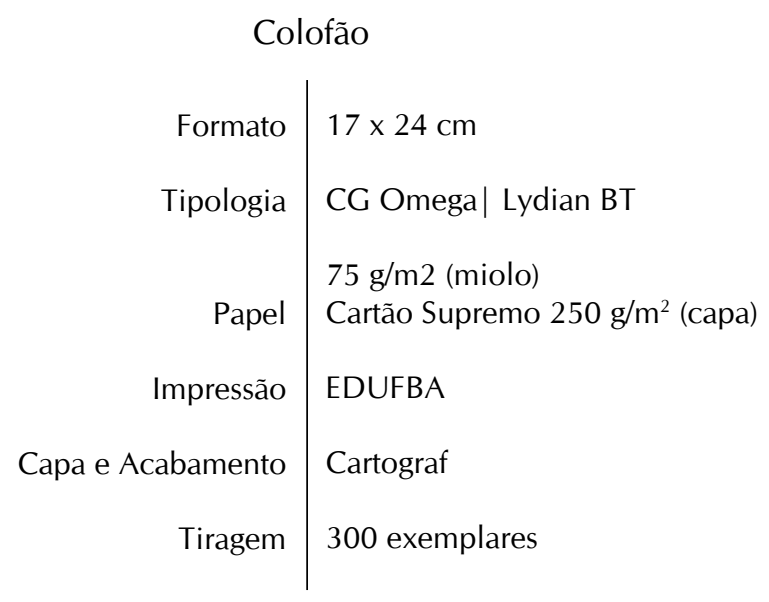

